\title{
Multiple Glomus Tumors of the Omentum
}

\author{
Won Beom Jung, In Ja Park, Joon Seon Song ${ }^{1}$, Kyung-Ja Cho ${ }^{1}$, Jin Cheon Kim \\ Departments of Colon and Rectal Surgery and ${ }^{1}$ Pathology, Asan Medical Center, University of Ulsan College of Medicine, Seoul, Korea
}

A glomus tumor is a very rare neoplasm consisting of cells that resemble the modified smooth muscle cells of normal glomus bodies. Here, we report a case of a 39-year-old male with multiple omental glomus tumors. The patient underwent a complete resection of the glomus tumors. This is a rare case of omental glomus tumors, and to our knowledge, this patient is the first with multiple omental glomus tumors to be described.

\section{Keywords: Multiple glomus tumor; Omentum}

\section{INTRODUCTION}

A glomus tumor is a rare, distinctive neoplasm consisting of cells that resemble the modified smooth muscle cells of normal glomus bodies and highly specialized glomic arteriovenous anastomoses that regulate temperature [1]. Although the incidence of glomus tumors is unclear, they are roughly estimated to constitute $<2 \%$ of all soft tissue tumors [2]. Glomus tumors are about equally common in both genders, although there is a striking female predominance (3:1) among patients with subungual lesions [3]. These usually occur in the acral soft tissue including that of the palm, wrist, forearm, and foot, with the single most common site being the subungual region of the fingers [4]. Glomus tumors are very rare in visceral locations, including the stomach, intestine, mediastinum, lung, pancreas, bladder, and vagina, with most intra-abdominal glomus tumors occurring in the stomach $[5,6]$. Clinically, glomus tumors have benign features, but occasionally show unusually large size, infiltrative growth, necrosis, nuclear atypia, or mitotic activity. Only a small number of glomus tumors have been diagnosed as malignant, with a diagnosis of an acral glomus tumor as malignant depending on the tumor's size and depth, the presence of atypical mitotic figures, and the tumor's

Received: April 18, 2015 - Accepted: June 6, 2015

Correspondence to: Jin Cheon Kim, M.D.

Department of Colon and Rectal Surgery, Asan Medical Center, University of Ulsan College of Medicine, 88 Olympic-ro 43-gil, Songpa-gu, Seoul 05505, Korea

Tel: +82-2-3010-3489, Fax: +82-2-474-9027

E-mail: jckim@amc.seoul.kr

(C) 2015 The Korean Society of Coloproctology

This is an open-access article distributed under the terms of the Creative Commons Attribution NonCommercial License (http://creativecommons.org/licenses/by-nc/3.0) which permits unrestricted noncommercial use, distribution, and reproduction in any medium, provided the original work is properly cited. nuclear grade [7].

This report describes a patient with multiple glomus tumors of the omentum. Two previous patients with omental glomus tumors have been reported in the English literature, one in Japan [6] and the other in India [8]. To our knowledge, this patient is the third reported with an omental glomus tumor and the first reported with multiple omental glomus tumors. Findings in this patient may help clinicians distinguish glomus tumors from other solid, soft-tissue tumors in the abdomen, including gastrointestinal stromal tumors and leiomyosarcomas.

\section{CASE REPORT}

A 39-year-old male presented with a recent onset of abdominal pain. No mass could be palpated on physical examination. Three years earlier, the patient had undergone a resection of a cerebellar hemangioblastoma, with no complications or sequelae. The patient's family history was not significant. Computed tomography (CT) revealed a solid and cystic mass with heterogeneous enhancement in the greater omentum near the anterior pelvis, with a focally enhanced nodule in the gallbladder bed (Fig. 1). The origin of the tumor could not be clearly determined. The lesion was separate from the bladder and the colon, and no evidence of metastasis to any solid organ was found. Magnetic resonance imaging (MRI) demonstrated two large masses with low signal intensity on T1-weighted images and high signal intensity on T2-weighted images (Fig. 2). The CT and the MRI results suggested a sarcoma of unknown origin, a mesothelioma, a hemangioma, or a desmoid tumor. A needle biopsy revealed that the tumor was an epithelioid mesenchymal neoplasm with hypervascularity.

The results of immunohistochemical (IHC) staining of the needle biopsy sample are shown in Table 1 . The Ki-67 labeling index 
was less than 5\%. The IHC results excluded the lesion from being a perivascular epithelioid cell tumor (PEComa), gastrointestinal stromal tumor, neuroendocrine carcinoma, adrenal cortical neoplasm, hepatocellular carcinoma, renal cell carcinoma, rhabdo- myosarcoma, desmoplastic round cell tumor, hemangioendothelioma, or epithelioid sarcoma. Based on its histology, the IHC results and size $(>10 \mathrm{~cm})$, a glomus tumor of uncertain malignant potential was highly suspected [7].
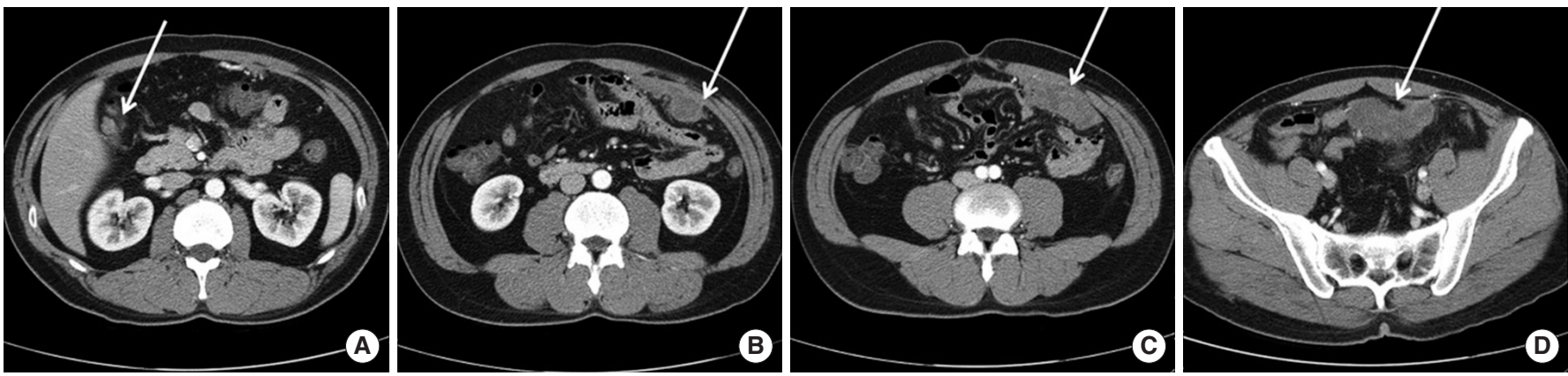

Fig. 1. Computed tomography scan findings. (A) A Focal enhancing nodule in the bed of the gallbladder. (B, C) Solid and cystic mass with heterogenous enhancement in the left omentum, (D) and anterior pelvis. The arrows point to the omental tumors.
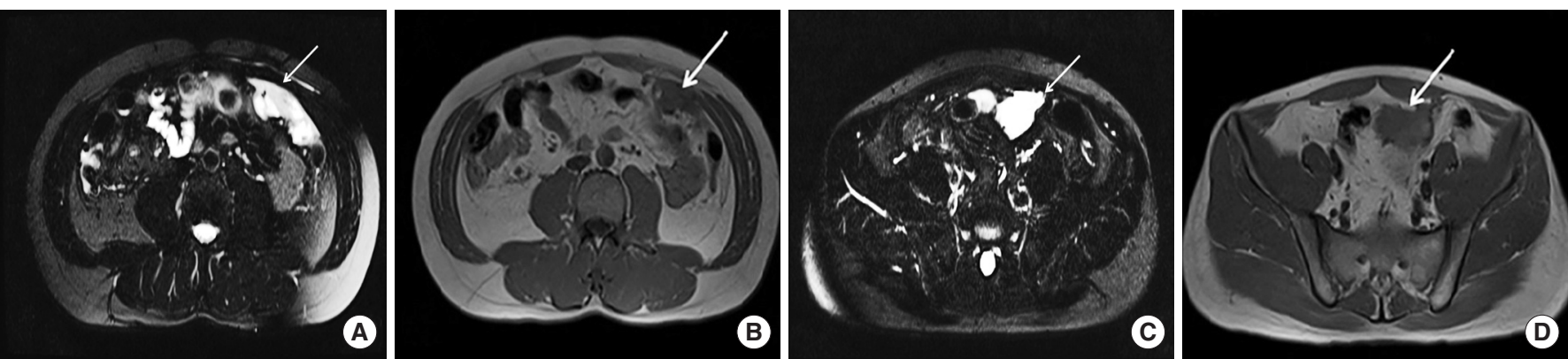

Fig. 2. Magnetic resonance imaging findings. Two large masses with high signal intensity on T2-WI in the left omentum (A) and pelvis (C), with low signal intensity on T1-WI in the left omentum (B) and pelvis (D). The arrows point to the omental tumors.

Table 1. Immunohistochemical staining of a needle aspiration biopsy sample obtained from the patient

\begin{tabular}{llllll}
\hline Positivity & \multicolumn{1}{c}{ Marker (antigen) } & \multicolumn{1}{c}{ Source } & \multicolumn{1}{c}{ Location } & \multicolumn{1}{c}{ Clone } & Dilution \\
\hline Focally (+) & Smooth muscle actin & Dako & Glostrup, Denmark & 1A4 & $1: 200$ \\
$(-)$ & Cytokeratin & Zymed & San Francisco, CA, USA & AE/AE3 & $1: 200$ \\
$(-)$ & S-100 & Zymed & San Francisco, CA, USA & poly & $1: 200$ \\
$(-)$ & CD117 & Dako & Glostrup, Denmark & poly & $1: 400$ \\
$(-)$ & TFE-3 & Cell Marque & Rocklin, CA, USA & MRQ-37 & $1: 100$ \\
$(-)$ & DOG-1 & Spring Bioscience & Pleasanton, CA, USA & SP31 & $1: 200$ \\
$(-)$ & Synaptophysin & Cell Marque & Rocklin, CA, USA & MRQ-40 & $1: 200$ \\
$(-)$ & Chromogranin & Dako & Glostrup, Denmark & DAK-A3 & $1: 200$ \\
$(-)$ & Desmin & Dako & Glostrup, Denmark & D33 & $1: 200$ \\
$(-)$ & Calretinin & Zymed & San Francisco, CA, USA & DC8 & $1: 400$ \\
$(-)$ & HMB45 & Dako & Glostrup, Denmark & HMB45 & $1: 50$ \\
$(-)$ & Inhibin & Serotec & Oxford, UK & R1 & $1: 100$ \\
$(-)$ & CD31 & Novo & Newcastle, UK & 1A10 & $1: 100$ \\
$(-)$ & CD34 & Immunotech & Marseille, France & QBEND10 & $1: 500$ \\
$(-)$ & PAX-8 & Cell Marque & Rocklin, CA, USA & Poly & $1: 100$ \\
$(-)$ & Hep par-1 & Dako & Glostrup, Denmark & OCH1E5 & $1: 200$ \\
\hline
\end{tabular}


Volume 31, Number 4, 2015

A median laparotomy was performed. Opening of the peritoneum revealed two large diffuse amorphous tumors attached to the greater omentum, one in the left upper quadrant of the abdominal cavity and the other in the pelvis. Additionally, one nodular, but amorphous, lesion attached to the lesser omentum was seen in the bed of the gallbladder. All tumors were completely resected. Although these tumors had not infiltrated into adjacent organs, whether these tumors had been completely resected microscopically was uncertain due to their diffuse and amorphous nature. The patient's recovery was uneventful.

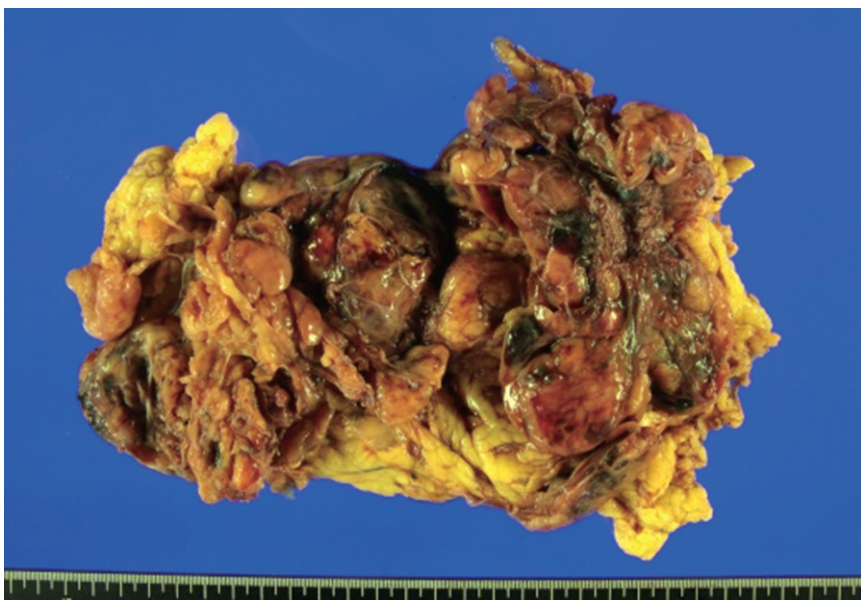

Fig. 3. Pathology results showing multiple solid masses measuring $13 \mathrm{~cm} \times 11 \mathrm{~cm} \times 9 \mathrm{~cm}$ in size.
The resected specimen appeared as a solid, disrupted mass with cystic changes and hemorrhage and measured $13 \mathrm{~cm} \times 11 \mathrm{~cm} \times 9$ $\mathrm{cm}$ in size. The cut surface of the mass was reddish brown, soft, and glistening with multifocal hemorrhage (Fig. 3). Microscopically, the tumor showed a diffuse solid sheet pattern with congestion. The tumor cells had epithelioid features, with round to oval nuclei and abundant eosinophilic cytoplasm. Some lymphocytes were scattered throughout the tumor. The borders of the tumor cells were clear. Multinucleated giant cells could occasionally be identified in the background of the dilated vessels. IHC of the resected specimen yielded results consistent with those of the needle biopsy sample. IHC with anti-CD34 antibody showed increased vascularity (Fig. 4). Mitotic activity was one per ten high-power fields. No necrosis was observed. These findings resulted in a final diagnosis of a glomus tumor of uncertain malignant potential. At the present time, four months after surgery, this patient continues to do well, with no complications and no evidence of recurrence.

\section{DISCUSSION}

Clinically, omental glomus tumors are difficult to diagnose because they are rare. A few retrospective studies reported that intra-abdominal glomus tumors occurred rarely, with no omental glomus tumor having been previously identified $[4,9]$. In addition, its symptoms, including abdominal pain, a palpable mass and intra-abdominal hemorrhage, are nonspecific $[6,8]$. Diagnosis, therefore, requires various radiologic techniques and IHC staining. Because glomus tumors are quite distinctive, owing to their characteristic cells and location, errors in diagnosis are infre-
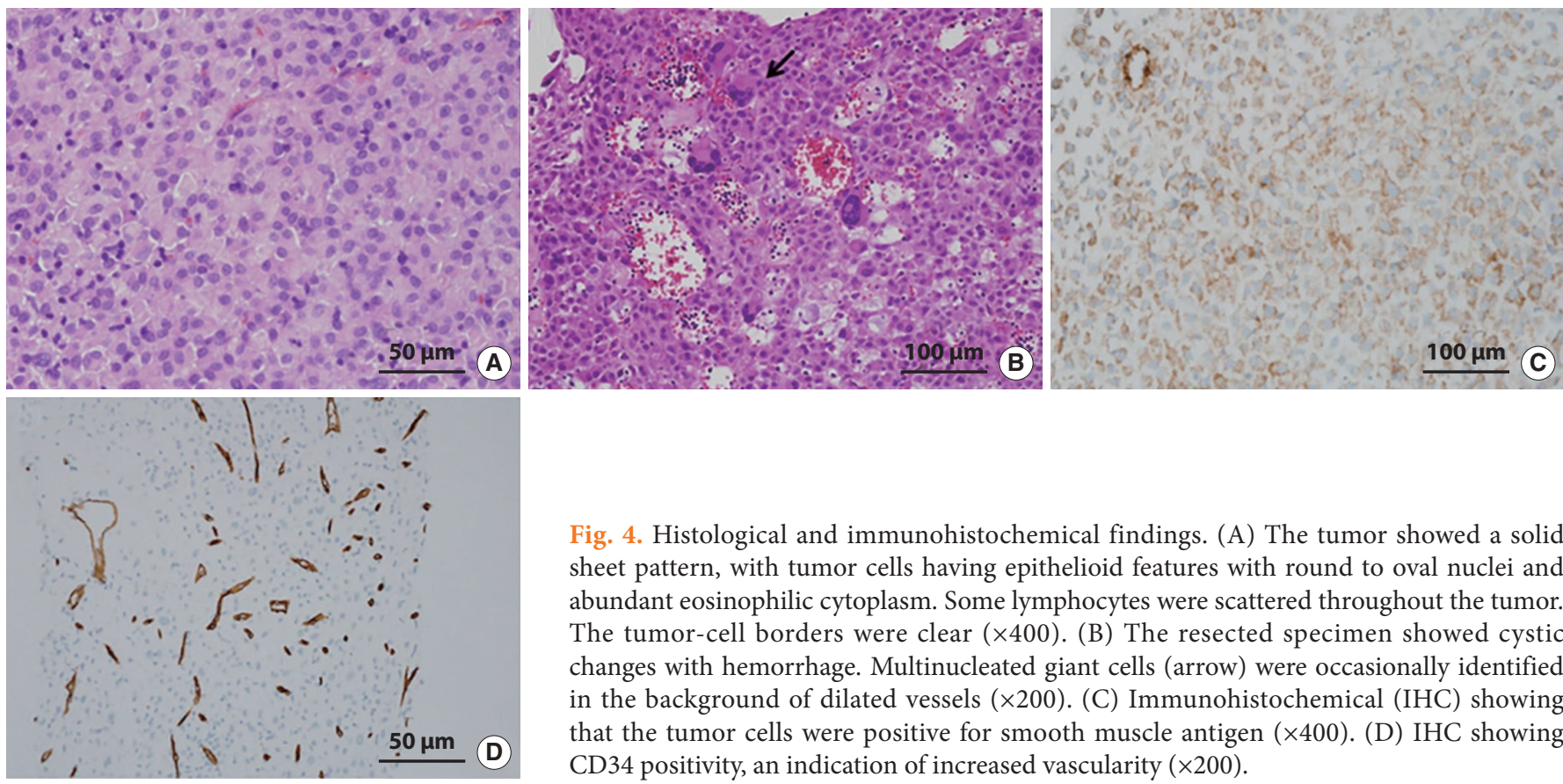

Fig. 4. Histological and immunohistochemical findings. (A) The tumor showed a solid sheet pattern, with tumor cells having epithelioid features with round to oval nuclei and abundant eosinophilic cytoplasm. Some lymphocytes were scattered throughout the tumor. The tumor-cell borders were clear $(\times 400)$. (B) The resected specimen showed cystic changes with hemorrhage. Multinucleated giant cells (arrow) were occasionally identified in the background of dilated vessels $(\times 200)$. (C) Immunohistochemical (IHC) showing that the tumor cells were positive for smooth muscle antigen $(\times 400)$. (D) IHC showing CD34 positivity, an indication of increased vascularity $(\times 200)$. 
quent after excision [10]. Although CT and MRI are useful in the diagnosis of intra-abdominal soft-tissue tumors, histologic examination is essential for an accurate diagnosis. Glomus tumors contain erythrocytes in simple tubes of endothelium, often surrounded by collagen sheaths. Moreover, tumor nodules, composed mainly of glomus-type epithelioid cells, may be present [11]. Histopathologically, these tumors appear as a sheet-like proliferation of oval or spindle-shaped cells exhibiting pale to eosinophilic cytoplasm around vessels and a lacework of basement membrane material around the cells. IHC staining shows that almost all glomus tumors are positive for vimentin and muscle actin isoforms, although desmin is highly variable, and heavy caldesmon may be present. Consistent with the ultrastructural features of these neoplasms, laminin and type-IV collagen, two constituents of basal lamina, outline the cells or small groups of cells [12]. The infiltrative component of the glomus tumor shows increases in the numbers of CD34 positive stromal cells [13].

Tumor excision is the treatment of choice. Local recurrences are infrequent, often representing tumor persistence following inadequate excision or a benign glomus tumor growing in a diffuse or infiltrative fashion [14]. As very few intra-abdominal glomus tumors have been described, their malignant potential remains uncertain. Although there is no evidence that these lesions are malignant or undergo malignant transformation, they may be difficult to eradicate completely. In one patient, microscopic residua of glomangiomatosis were associated with persistence of pain, and a wide excision was required to alleviate the symptoms [15].

Classic glomus tumors are typically solitary. To our knowledge, this patient is the first with multiple omental glomus tumors to be described. Intra-abdominal glomus tumors have been misdiagnosed as gastrointestinal stromal tumors, leiomyosarcomas, or leiomyomas. Although the radiologic results in our patient were not indicative of a glomus tumor, needle aspiration biopsy results were consistent with the final diagnosis. Thus, needle aspiration biopsy, followed by IHC, may be helpful in the diagnosis of intraabdominal soft-tissue tumors. The tumors in this patient were diffuse and multiple and could not be palpated, suggesting that microscopic residua may have remained after excision. IHC staining showed positivity for $\mathrm{CD} 34$, which may be associated with an infiltrative component. These findings indicate the need for periodic follow-up for this patient by using imaging methods and assessments of symptoms as surveillance for tumor recurrence.

\section{CONFLICT OF INTEREST}

No potential conflict of interest relevant to this article was reported.

\section{REFERENCES}

1. Tsuneyoshi M, Enjoji M. Glomus tumor: a clinicopathologic and electron microscopic study. Cancer 1982;50:1601-7.

2. Fletcher CD, Unni KK, Mertens F. Pathology and genetics of tumours of soft tissue and bone. Lyon: IARC; 2002.

3. Takata H, Ikuta Y, Ishida O, Kimori K. Treatment of subungual glomus tumour. Hand Surg 2001;6:25-7.

4. Enzinger FM, Weiss SW. Soft tissue tumors. St. Louis: CV Mosby; 1988.

5. Lee HW, Lee JJ, Yang DH, Lee BH. A clinicopathologic study of glomus tumor of the stomach. J Clin Gastroenterol 2006;40:71720.

6. Matsuo S, Susumu S, Tsutsumi R, Azuma T, Obata S, Hayashi T. Glomus tumor of the omentum: a case report. J Surg Oncol 2007; 96:633-6.

7. Folpe AL, Fanburg-Smith JC, Miettinen M, Weiss SW. Atypical and malignant glomus tumors: analysis of 52 cases, with a proposal for the reclassification of glomus tumors. Am J Surg Pathol 2001; 25:1-12.

8. Alam K, Maheshwari V, Sabir F, Haq ME, Siddiqui FA, Mefuzuddin S. Glomus tumor of lesser omentum: a case report. Indian J Pathol Microbiol 2007;50:543-4.

9. Heys SD, Brittenden J, Atkinson P, Eremin O. Glomus tumour: an analysis of 43 patients and review of the literature. Br J Surg 1992; 79:345-7.

10. Rao VK, Weiss SW. Angiomatosis of soft tissue. An analysis of the histologic features and clinical outcome in 51 cases. Am J Surg Pathol 1992;16:764-71.

11. Murray MR, Stout AP. The glomus tumor: investigation of its distribution and behavior, and the identity of its "epithelioid" cell. Am J Pathol 1942;18:183-203.

12. Haque S, Modlin IM, West AB. Multiple glomus tumors of the stomach with intravascular spread. Am J Surg Pathol 1992;16:291-9.

13. Skelton HG, Smith KJ. Infiltrative glomus tumor arising from a benign glomus tumor: a distinctive immunohistochemical pattern in the infiltrative component. Am J Dermatopathol 1999;21:562-6.

14. Gould EW, Manivel JC, Albores-Saavedra J, Monforte H. Locally infiltrative glomus tumors and glomangiosarcomas. A clinical, ultrastructural, and immunohistochemical study. Cancer 1990;65: 310-8.

15. Ezeoke C, Xiang D, Poddar N. A rare case of glomus tumor of the thigh: malignant or not? Int J Case Rep Images 2014;5:818-21. 